\title{
Field Induced Unusual Magnetic Behavior at Low Temperature in $\mathrm{Pr}_{0.67} \mathrm{Ca}_{0.33} \mathrm{MnO}_{3}$
}

\author{
M. Pektas*, H. Gencer, T. Izgi, V.S. Kolat and S. Atalay \\ Inonu University, Science and Arts Faculty, Physics Department, 44069 Malatya, Turkey
}

\begin{abstract}
In this study, magnetic properties of $\operatorname{Pr}_{0.67} \mathrm{Ca}_{0.33} \mathrm{MnO}_{3}$ compound were investigated in detail. The magnetization versus temperature $(M-T)$ curve showed that this material undergoes a charge order transition at $200 \mathrm{~K}$. A more pronounced FM phase appeared below $56 \mathrm{~K}$. At $T=5 \mathrm{~K}$, a field induced sharp step like magnetisation transition associated with phase separation was observed. However, after application of $7 \mathrm{~T}$ magnetic field at $5 \mathrm{~K}$, the magnetic behaviour of sample was changed completely and full FM behaviour was observed. The more interesting is that the sample remains in the FM state and does not retrieve the initial magnetic state until warmed up to charge order transition temperature. Large negative magnetic entropy change $(-26.18 \mathrm{~J} /(\mathrm{kg} \mathrm{K})$ at $38 \mathrm{~K}$ and $5 \mathrm{~T}$ ) was attributed to step like magnetisation transition.
\end{abstract}

DOI: $10.12693 /$ APhysPolA.125.217

PACS: 75.47.Lx, 75.30.Kz, 75.30.Sg

\section{Introduction}

Mixed valence perovskite manganites with the general formula $\mathrm{R}_{1-x} \mathrm{~A}_{x} \mathrm{MnO}_{3}$ have fascinating rich properties and magnetic phases resulting from the strong coupling between the charges, spin, orbital, and lattice degrees of freedom. Due to the subtle balance between various phases, some manganites display particularly rich and unusual behaviors as in the case of $\mathrm{Pr}_{0.67} \mathrm{Ca}_{0.33} \mathrm{MnO}_{3}$ compound. Earlier experimental studies have shown that upon cooling from high temperature in low fields, this compound undergoes a charge order $(\mathrm{CO})$ transition at $T_{\mathrm{CO}}$ followed by antiferromagnetic (AFM) and ferromagnetic (FM) transitions [1]. In some recent papers, the neutron scattering studies have shown that the low temperature ground state of this compound is inhomogeneous with coexistence of submicrometer FM metallic and $\mathrm{CO}-\mathrm{AFM}$ insulating regions which is called phase separation (PS) [2]. Interestingly, a chemically homogeneous material forms a magnetically inhomogeneous system with spatially coexisting regions with distinct magnetic and electronic properties. The competition between both phases is resolved in a nanoscale length, giving rise to real-space inhomogeneities in the material. It is also now well understood that PS state in manganites is close related with the competition between CO-AFM insulating and FM metallic phases.

Another surprising result found in $\mathrm{Pr}_{0.67} \mathrm{Ca}_{0.33} \mathrm{MnO}_{3}$ compound is the appearance of ultrasharp magnetization steps at low temperatures [3]. This ultrasharp magnetic transition is included in the category of metamagnetic transitions which is defined as the field induced transition of the entire compound from one phase to the other of PS state. However, unlike the broad continuous transitions expected for inhomogeneous granular systems, in

*corresponding author; e-mail: melike_pektas_87@hotmail.com this case transition occurs in an extremely narrow magnetic fields range and at low temperature. These ultrasharp metamagnetic transitions were observed in single crystal, polycrystalline, and thin film forms of manganites, indicating that it is mainly an intrinsic nature of the samples [4].

Among various mixed valence manganites studied so far, $\mathrm{Pr}_{0.67} \mathrm{Ca}_{0.33} \mathrm{MnO}_{3}$ is of great interest for the study of the PS and consequently the metamagnetic transition. Due to the coexistence of AFM-CO and FM metallic phases in this compound, the system provides an opportunity to study these phase competition phenomena and ultrasharp metamagnetic phase transition. In this work, we report magnetic properties of $\mathrm{Pr}_{0.67} \mathrm{Ca}_{0.33} \mathrm{MnO}_{3}$ compound as a function of temperature and magnetic field. At low temperature, field induced unusual irreversible ferromagnetic transition is investigated in detail.

\section{Experimental}

The polycrystalline $\operatorname{Pr}_{0.67} \mathrm{Ca}_{0.33} \mathrm{MnO}_{3}$ compound was prepared by the conventional solid-state reaction using high purity powders $\operatorname{Pr}_{6} \mathrm{O}_{11}, \mathrm{CaCO}_{3}, \mathrm{MnO}$. The powders were mixed in stoichiometric ratio. The X-ray diffractograms were recorded with a power diffractometer at room temperature using $\mathrm{Cu} K_{\alpha}$ radiation. Grain structure was observed using a LEO-EVO-40 scanning electron microscope. The magnetic measurements were performed using a Q-3398 (Cryogenic) magnetometer in a temperature range from 5 to $300 \mathrm{~K}$ and $6 \mathrm{~T}$ maximum magnetic field was applied.

\section{Results and discussion}

Figure 1a shows the temperature dependence of the magnetization of $\operatorname{Pr}_{0.67} \mathrm{Ca}_{0.33} \mathrm{MnO}_{3}$ compound. The data were taken under an applied field of $0.1 \mathrm{~T}$ after the sample was zero-field cooled (ZFC) and field-cooled (FC). Both the ZFC and FC curves show an anomaly around $200 \mathrm{~K}$ (marked as $T_{\mathrm{CO}}$ ). The observed broad hump around $200 \mathrm{~K}$ was attributed to $\mathrm{CO}$ state. At about $140 \mathrm{~K}$ (marked as $T_{\mathrm{AFM}}$ ), a weak but noticeable maximum is also observed, which indicates the onset of the 
AFM ordering. At lower temperatures, the sample displays upturn in magnetization at about $110 \mathrm{~K}$ corresponding to a rapid growth of FM ordering in the CO-AFM matrix. A more pronounced FM phase appears at $T_{\mathrm{c}}=56 \mathrm{~K}$. Below about $40 \mathrm{~K}$, the magnetization shows a splitting between the ZFC and FC curves which indicates the existence of a spin-glass like behavior. The magnetization for $\mathrm{FC}$ has a larger value than that of the ZFC suggesting that applied field favors the formation of FM phase compared with CO-AFM one. As can be seen in Fig. 1a, when the temperature is increased from low temperature, the magnetization exhibits a small increase up to $18 \mathrm{~K}$. Although this feature is not entirely understood, in many studies it has been interpreted as due to the thermally induced increase in FM phase fraction. The sample is blocked in a metastable PS state due to the disorder or the strains between the FM and CO-AFM states. With the increase in temperature, the system becomes unblocked due to the thermal fluctuation and the magnetization shows a small increase $[5,6]$.
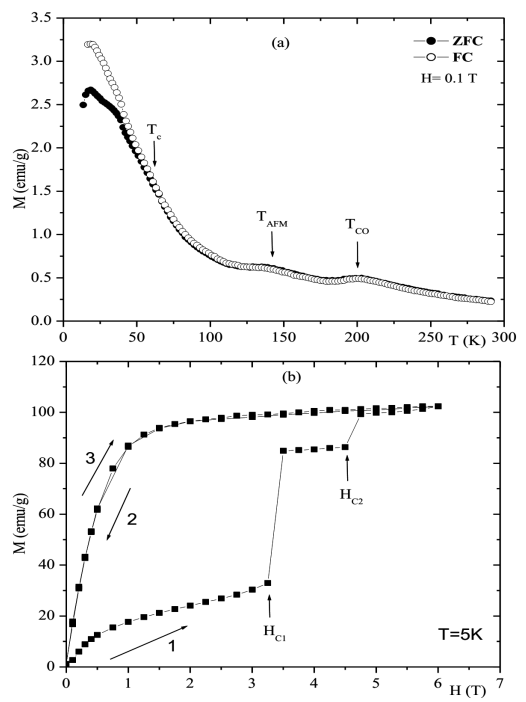

Fig. 1. (a) The temperature dependence of magnetization of $\mathrm{Pr}_{0.67} \mathrm{Ca}_{0.33} \mathrm{MnO}_{3}$ compound measured warming after ZFC and FC in a field of $0.1 \mathrm{~T}$. (b) Isothermal magnetization curve measured at $5 \mathrm{~K}$ for $\operatorname{Pr}_{0.67} \mathrm{Ca}_{0.33} \mathrm{MnO}_{3}$ compound.

Figure $1 \mathrm{~b}$ shows the magnetization measurement as a function of magnetic field of $\operatorname{Pr}_{0.67} \mathrm{Ca}_{0.33} \mathrm{MnO}_{3}$ compound at $5 \mathrm{~K}$. A sudden increase of magnetization was observed at a critical field $\left(H_{\mathrm{C}}\right)$. The appearance of a sharp step-like transition, which is called as metamagnetic transition, is a clear signature of the coexistence of FM and CO-AFM phases. The origin of this step-like transition is still a matter of controversy. Different interpretations have been proposed [2-7]. In most of interpretations the step-like transition in manganites is based on the scenario of PS, in which the CO-AFM and FM phases coexist in the sample and FM phase develops in the CO-AFM matrix. There is a considerable competition between these two phases. The slightly different cell parameter of the FM and $\mathrm{CO}$ phases generates strain at the interface region. Although the FM phase starts to develop below CO temperature, at low temperatures its growth is impeded by interface strain. Consequently, the FM cluster cannot grow against the CO-AFM background. At that point application of a magnetic field lowers the energy of the FM phase. As the magnetic field is large enough for the deriving force action on the spins to overcome the constraints, the local stress is destabilized, which causes a sudden jump in magnetization [2, 3].

Depending on the strength of the magnetic field, the volume ratio of CO-AFM phase changes to FM phase. As the magnetic field is increased enough, all the regions behave as CO-AFM matrix is converted into an almost full FM state and magnetization reaches almost saturation. However, when the magnetic field is decreased from high value to zero a large irreversibility in magnetization was observed (labeled as path 2 in Fig. 1b). For the further magnetic field application, the sample remains in the last acquired magnetic state (labeled as path 3 in Fig. 1b) and do not retrieve the initial magnetic state. Additionally, Wu et al. [3] have concluded that the number of steps observed on $M(H)$ curve (Fig. 1b) strongly depends on the details of the synthesis and measurement procedure and the thermal and magnetic history of the sample.

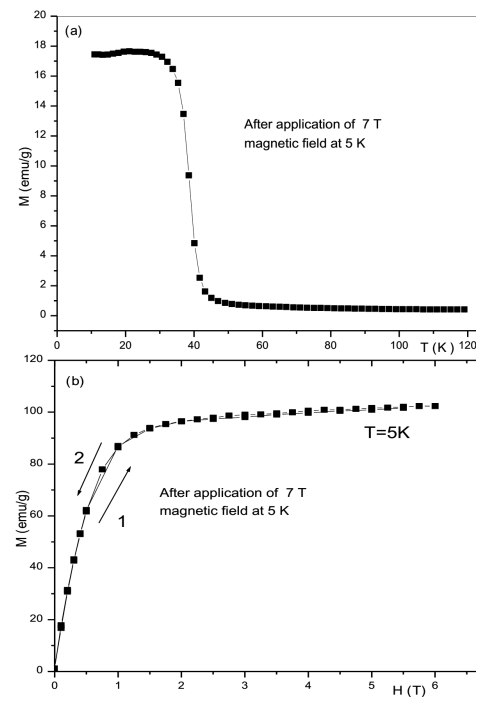

Fig. 2. (a) The temperature dependence of magnetization of $\mathrm{Pr}_{0.67} \mathrm{Ca}_{0.33} \mathrm{MnO}_{3}$ compound after application of $7 \mathrm{~T}$ magnetic field at $5 \mathrm{~K}$ and (b) isothermal magnetization curve measured at $5 \mathrm{~K}$ after application of $7 \mathrm{~T}$ magnetic field.

In order to clarify the irreversible magnetization transition, magnetic measurements of the sample were repeated after application of $7 \mathrm{~T}$ magnetic field at $5 \mathrm{~K}$. The results are given in Fig. $2 \mathrm{a}$ and $\mathrm{b}$. If the results given in Fig. 1 are compared with Fig. 2, it could be clearly seen that the magnetic behaviors of the sample was changed completely. The results given in Fig. 2 have indicated that the sample shows fully FM behavior. Interestingly, once the magnetic field is applied the sample 
remains in the FM state and does not retrieve the initial PS mixed magnetic state even after magnetic field is removed. The previous magnetic behaviors of the sample are not observed unless the sample is heated up above $\mathrm{CO}$ transition temperature. We have seen that when the sample is heated up to CO transition temperature, the effect of magnetic field disappeared and the sample showed again the previous magnetic behaviors in Fig. 1. As concluded above, due to the strain energy between FM and CO-AFM phases, the mixed phase state has higher energy and the system is not stable. The application of $7 \mathrm{~T}$ magnetic field, which is large enough to overcome the strain energy between FM and CO-AFM phases, lowers the energy of FM phase and the system has full FM ordering. Roy et al. [1] have concluded that if the system undergoes a metamagnetic phase transition, then the free energy of the system changes continuously. In such cases, there is an energy barrier to the nucleation of the equilibrium phase that is typically characterized as a positive surface energy of the interface between the CO-AFM and FM phases. Once the system is in the FM state, there is no energy advantage to reverting to the CO-AFM state even after the external magnetic field is removed unless the temperature reached up to $\mathrm{CO}$ transition temperature. Above CO transition temperature, such a thermal energy is sufficient to overcome the FM phase due to the thermal fluctuation and sample shows again the previous magnetic behaviors.

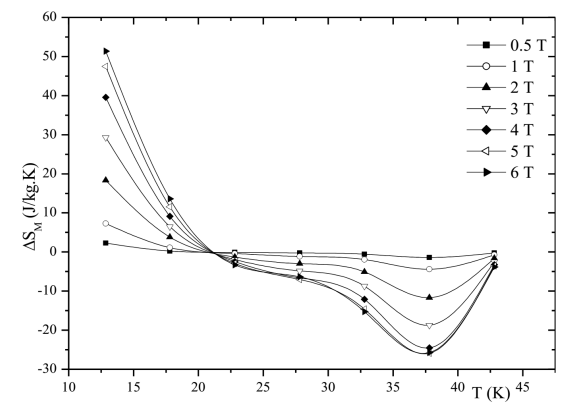

Fig. 3. Magnetic entropy change of $\operatorname{Pr}_{0.67} \mathrm{Ca}_{0.33} \mathrm{MnO}_{3}$ sample at low temperatures.

The magnetic entropy, which is associated with the MC effect, can be calculated from the isothermal magnetization curves (which are not given here) under the influence of a magnetic field. According to the classical thermodynamical theory, the magnetic entropy change $\Delta S_{\mathrm{m}}$ produced by the variation of a magnetic field from 0 to $H_{\text {max }}$ is given by

$$
\Delta S_{\mathrm{m}}(T, H)=\int_{0}^{H_{\max }}\left(\frac{\partial M}{\partial T}\right)_{H} \mathrm{~d} H .
$$

To evaluate the magnetic entropy change $\Delta S_{\mathrm{m}}$, a numerical approximation of the integral in Eq. (1) and the experimental $M-H$ curves at various temperatures have been used. Figure 3 shows the magnetic entropy change as a function of temperature at various magnetic field for $\operatorname{Pr}_{0.67} \mathrm{Ca}_{0.33} \mathrm{MnO}_{3}$ sample at low temperatures. As can be seen in Fig. 3, at just below $40 \mathrm{~K}$ we find extremely large value of $\Delta S_{\mathrm{m}}$. The presence of FM regions dispersed in the CO-AFM matrix leads to a magnetization increase with decreasing temperature just below $110 \mathrm{~K}$ and then reaches the maximum value at near $40 \mathrm{~K}$. In this temperature range, due to the presence of FM and CO-AFM phases, application of magnetic field causes a metamagnetic transition. As a consequence of metamagnetic transition, the magnetic entropy change $\left(\Delta S_{\mathrm{m}}\right)$ reaches $-26.18 \mathrm{~J} /(\mathrm{kg} \mathrm{K})$ at $38 \mathrm{~K}$ and $5 \mathrm{~T}$ magnetic field. At still lower temperature $(T<22 \mathrm{~K}) \Delta S_{\mathrm{m}}$ becomes positive.

\section{Conclusions}

In this study, magnetic properties of $\operatorname{Pr}_{0.67} \mathrm{Ca}_{0.33} \mathrm{MnO}_{3}$ compound were investigated in detail. The magnetization curves showed a weak maximum around $200 \mathrm{~K}$, which is a signature of the $\mathrm{CO}$ transition. At about $140 \mathrm{~K}$, a weak maximum was attributed to onset of the AFM ordering. At lower temperatures, the sample displayed upturn in magnetization at about $110 \mathrm{~K}$ corresponding to a rapid growth of FM ordering. A more pronounced FM phase appeared at $T_{\mathrm{c}}=56 \mathrm{~K}$. At $T=5 \mathrm{~K}$, the magnetization isotherm showed two sharp step-like metamagnetic transition at 3.2 and $4.5 \mathrm{~T}$. This sharp step-like transition was attributed to coexistence of FM and CO-AFM phases at low temperatures. However, after application of $7 \mathrm{~T}$ magnetic field at $5 \mathrm{~K}$, the magnetic behavior of sample was changed completely. The results showed that the sample has a full FM behavior. The more interesting is that the sample remains in the FM state and does not retrieve the initial PS mixed magnetic state unless the temperature reached up to CO transition temperature. At lower temperatures, observed extremely large negative magnetic entropy change $(-26.18 \mathrm{~J} /(\mathrm{kg} \mathrm{K})$ at $38 \mathrm{~K}$ and $5 \mathrm{~T}$ ) was attributed to ultrasharp metamagnetic transitions.

\section{Acknowledgments}

This work was supported by Inonu University Research Fund with the project number 2012/170.

\section{References}

[1] M. Roy, J.F. Mitchell, P. Ramirez, P. Schiffer, Philos. Mag. 81, 417 (2001).

[2] R. Mathieu, Y. Tokura, J. Phys. Soc. Jpn. 76 , 124706 (2007).

[3] T. Wu, J.F. Mitchell, J. Magn. Magn. Mater. 292 , 25 (2005).

[4] M.H. Phan, S.C. Yu, J. Magn. Magn. Mater. 308, 325 (2007).

[5] V.S. Kolat, T. Izgi, A.O. Kaya, N. Bayri, H. Gencer, S. Atalay, J. Magn. Magn. Mater. 322, 427 (2010).

[6] S. Mollah, H.L. Huang, S.J. Liu, P.L. Ho, W.L. Huang, C.W. Huang, C.P. Sun, J.-Y. Lin, Y.S. Gou, W.-H. Li, H.D. Yang, J. Magn. Magn. Mater. 265, 215 (2003).

[7] K.R. Mavani, P.L. Paulose, Europhys. Lett. 78, 37004 (2007). 